\title{
OCULTACIÓN DE LA RIQUEZA RÚSTICA EN ESPAÑA (1870-1936): ACERCA DE LA FIABILIDAD DE LAS ESTADÍSTICAS SOBRE LA PROPIEDAD Y USO DE LA TIERRA
}

\author{
JUAN PRO RUIZ \\ Universidad Autónoma de Madrid
}

\section{RESUMEN}

La Contribución territorial, creada en 1845 , estaba viciada por la carencia de un sistema eficaz de obtención de datos sobre la propiedad y uso de la tierra. Los documentos conocidos como «amillaramientos» no sólo carecen de fiabilidad por contener un alto nivel de ocultación, sino que en realidad eran fruto de negociaciones políticas y no de averiguaciones estadísticas. El análisis pormenorizado del caso de la provincia de Cádiz confirma este punto de vista. En consecuencia, la imagen del crecimiento agrícola español de la segunda mitad del siglo XIX y del primer tercio del XX, debería ser matizada con el hecho de que coincide con un periodo de mejora en la calidad de los datos oficiales sobre superficie cultivada, producciones y rendimientos: de hecho, las series que venimos manejando parecen partir de niveles artificialmente bajos, en virtud de la prevención antifiscal dominante en el mundo rural de entonces.

\section{ABSTRACT}

The Land Tax, established in Spain in 1845, suffered from the lack of an efficient system of data collection concerning ownership and the use of land. The documents known as «amillaramientos" are unworthy of trust, not only due to their high level of concealment, but also as result of the fact that they were by nature the fruits of political negociations and not of statistical research. A detailed analysis of the province of Cádiz confirms this hypothesis. As a result, the study of the Spanish agricultural growth of the second half of the nineteenth century and the first third of the twentieth century must consider the coincidence between this time period and the growing improvement in the quality of the official information concerned with cultivated areas, productions and profits. The series so far used depart, from artificially low figures, distorted by the antifiscal efforts which dominated the Spanish rural world at the time. 
El término específico con el que se designaban en el siglo XIx las operaciones de evasión fiscal era el de ocultación. Dicho término, hoy caido en desuso, significa la acción de encubrir bienes con el fin de no pagar tributos; es, por lo tanto, un término meramente descriptivo y muy adecuado a las caracteristicas de un impuesto de producto (como era la Contribución territorial), en el que la ocultación de la cosa traía aparejada la inexistencia del tributo. La ocultación fue definida expresamente por el Reglamento de amillaramientos de 1878 , al tiempo que se declaraba que podía ser objeto de denuncia, investigación y penalidad. Entraban en tal consideración casos diversos: por un lado, la omisión en las declaraciones de fincas o cabezas de ganado, o bien la disminución de la superficie de los inmuebles (dos casos de ocultación absoluta); y por otro, la desnaturalización de la clase de cultivo a la que se dedicaban las fincas, clasificándolas en otras de menor rendimiento, la inferioridad en clase o edad del ganado, y la declaración de una renta menor de la real en aquellas fincas que estuvieran arrendadas (tres supuestos de ocultación relativa). La actitud pasiva de los propietarios que admitieran errores a su favor era conceptuada también como ocultación ${ }^{1}$.

\section{AMILLARAMIENTOS: EL FRAUDE INSTITUCIONALIZADO}

El nuevo sistema tributario creado por la reforma de Mon (1845) se basaba en varios impuestos de los llamados «de producto», el más importante de los cuales era la Contribución de inmuebles, cultivo y ganadería. Para repartir los cupos de dicho impuesto se recurrió, desde 1850 , a realizar en cada pueblo unos amillaramientos (listas de propiedades de los vecinos) y unas cartillas evaluatorias (cálculo del rendimiento normal de los diferentes usos y calidades del suelo de la localidad). Tales documentos los realizaban unas juntas periciales formadas por los miembros del ayuntamiento y un número igual de los mayores contribuyentes; la Administración central se limitaba a negociar con estas juntas locales el cupo del tributo, y les dejaba las manos libres respecto al reparto de su pago entre los vecinos. Al no existir documentos gráficos que reflejaran objetivamente la propiedad y uso de la tierra, la ocultación era sistemática y el fraude proliferaba con total impunidad.

La época de confección de los amillaramientos y de su posterior contrastación con el catastro coincidió, además, con el proceso de introducción en Es-

1 Capitulo VIII del R.D. de 10 de diciembre de 1878, publicando el Reglamento sobre rectificación de amillaramientos (Colección Legislativa, 1879, pp. 25-165). 
paña de las unidades de medida del sistema métrico decimal, lo cual introdujo un elemento adicional de confusión y, por ende, adicionales oportunidades para la ocultación. Los amillaramientos respondían a un modelo de documento utilizado en España desde tiempo inmemorial para repartir los impuestos: eran listas de propietarios de un pueblo, a los cuales se asignaban una tras otra sus distintas propiedades. La descripción de los predios se hacía trasladando al papel la información que proporcionaban los vecinos en un estilo propio de la lengua hablada: se relacionaban los nombres de los propietarios colindantes a los cuatro vientos, de tal manera que resultaba de todo punto imposible reconstruir el parcelario para comprobar si habia fincas no declaradas. Tal descripción se completaba con una evaluación aproximada de la superficie del predio, bajo la forma tradicional de la «cabida», es decir, indicando cuánta semilla podía sembrarse en un terreno determinado. Con el tiempo, aquellas unidades de medida basadas en la siembra habian cristalizado en el lenguaje de la gente del campo en unidades de superficie propiamente dichas, que, bajo el mismo nombre, recubren una variedad enorme de extensiones reales.

Desde 1849 el gobierno empezó a introducir en el mundo rural el sistema métrico decimal, eliminando la heterogeneidad de unidades existente 2 ; la tarea se extendió durante más de cincuenta años, pues hasta el primer tercio del siglo $\mathrm{xx}$ no empezaron a utilizarse áreas y hectáreas como unidades de medida en el campo español. Pero, incapaz de abarcar la variedad de unidades que se utilizaban durante la segunda mitad del XIX, la Administración empleó algunos supuestos simplificadores, como el de atribuir a cada provincia una equivalencia en hectáreas de la fanega o de la unidad de medida más usada; de hecho, cada pueblo o grupo de pueblos tenía sus unidades particulares, y a veces hasta dentro de un mismo pueblo se utilizaban distintas unidades de medida para las tierras llanas y las tierras altas, o para la viña y el cereal, o para el regadío y el secano ${ }^{3}$. Y, sobre todo, lo que hacía ilusorios los intentos de cálculo racional, era el hecho de que las unidades de medida tradicionales, reflejadas en los documentos fiscales, no tenían pretensiones de exactitud, sino que respondían a una impresión personal, influenciada por la calidad de los terrenos, su orografía y su vegetación ${ }^{4}$. Si a esto unimos que muchos propietarios no habían realizado nunca una medición rigurosa de sus fincas, comprendemos que a la ocultación deliberada para eludir la carga del impuesto se añadía la indefinición propia del mundo rural de aquella época.

2 Tablas de correspondencia... (1852); Instituto Geográfico y Estadístico (1886).

3 Lopera (1958) y Besnier (1964).

4 Pro (1994a). 
La amplitud de la riqueza territorial que se ocultaba a la Hacienda no empezaría a conocerse sino veinte años después de que se creara el sistema de los amillaramientos; la llegada al poder de progresistas y demócratas tras la Revolución de 1868 sacó a la luz pública datos que demostraban hasta qué punto la práctica tributaria se había alejado de los principios teóricos de equidad que inspiraban el sistema.

Cuando Laureano Figuerola se hizo cargo del Ministerio de Hacienda -en octubre de 1868- sólo encontró en sus oficinas centrales los resúmenes de los amillaramientos de 23 provincias, de modo que tuvo que tomarse el trabajo de conseguir los restantes y de reducirlos todos a unidades de medida del sistema métrico decimal (no consiguió datos relativos a Álava, Guipúzcoa, Vizcaya y Navarra, por razón de los privilegios fiscales que mantenian; pero tampoco para La Coruña y Pontevedra, provincias en las que ni siquiera parecen haberse elaborado amillaramientos 5 ). A continuación comparó las cifras de aquellos documentos con la superficie del pais y de sus diferentes provincias, lo cual le permitió ofrecer a la opinión pública la primera imagen oficial de la magnitud del fraude fiscal de los terratenientes (mapa 1).

El impacto de semejantes datos, lanzados desde el propio ministerio, resulta innegable: 25 años después de la reforma liberal de la Hacienda, 18 millones de hectáreas -más de un 39 por 100 de la superficie rústica del pais- no estaban recogidas en los amillaramientos, y en 13 provincias la extensión de tierra oculta era mayor que la declarada.

Las cifras publicadas por el ministerio en 1870 pretendían exponer ante la sociedad española el problema del fraude. $\mathrm{Al}$ mismo tiempo, se pretendía quizá asustar a los defraudadores con la perspectiva de una mayor presión social sobre ellos y de una mayor voluntad política de perseguirles desde la Administración del Estado. Aparte de esas intenciones «simbólicas», aquellos datos tenían poco valor, ya que no existía aún el catastro que hubiera permitido comprobarlos 6: tan sólo tenian el valor de ser los primeros datos oficiales de carácter general y el de indicarnos dónde pensaba el gobierno que se situaban

5 Figuerola consiguió estimaciones fiscales de la riqueza territorial para las otras dos provincias gallegas y para la de Oviedo; pero, en realidad, en la segunda mitad del xIX no se elaboraron amillaramientos sistemáticos ni en Galicia ni en Asturias, de modo que en aquellos territorios los repartos de la Contribución territorial se realizaban con la más amplia libertad de movimientos por parte de los ayuntamientos y juntas periciales, pues ni siquiera elaboraban un documento que pudiera ser objeto de reclamación.

- A la altura de 1870 sólo se habian realizado levantamientos catastrales en algunos pueblos de Castilla la Nueva (Guadalajara, Toledo y Cuenca), en los que se venía trabajando desde 1867; por cierto, que ya en aquel primer acercamiento se comprobó una ocultación media del 40 por 100 de las superficies (Sociedad Económica Matritense de Amigos del Pais, 1874, pp. 5-6). 


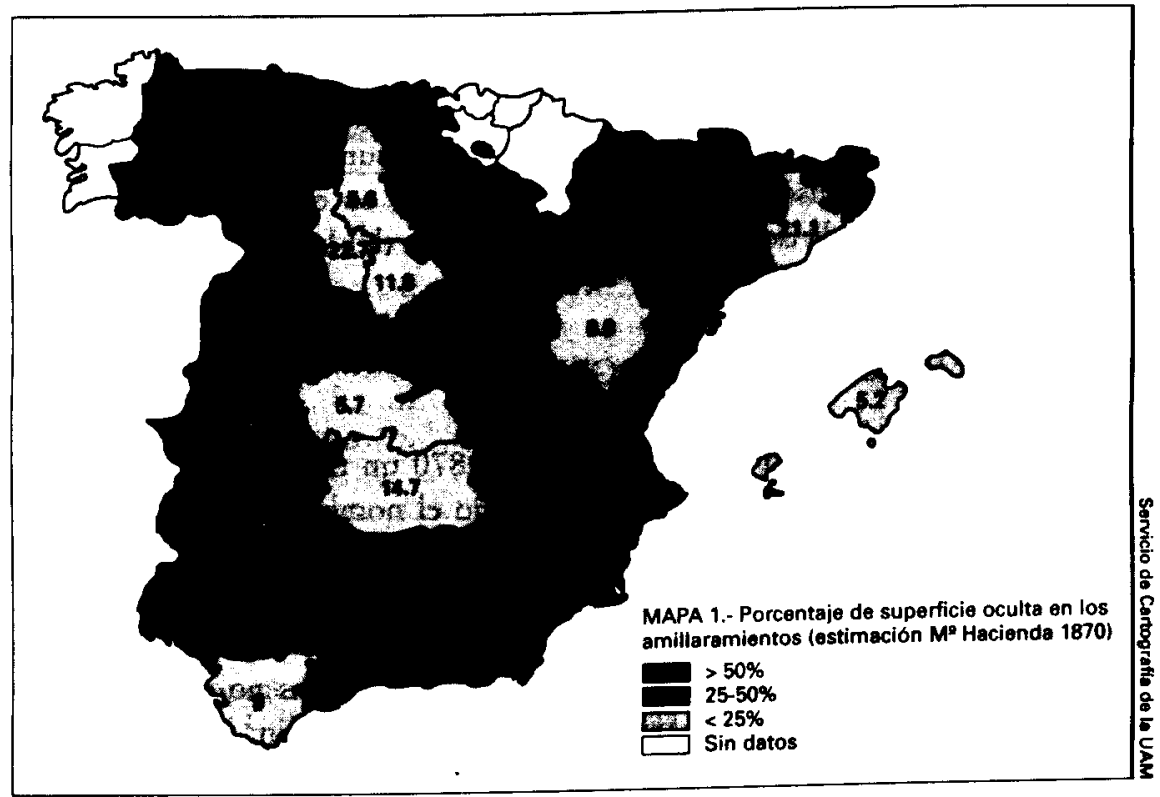

FuenTE: Garcia de Torres (1872), pp. 168-169.

las mayores bolsas de ocultación. A ese respecto, creo que los datos de 1870 pudieron ser contraproducentes, por cuanto, si observamos la geografía del fraude que nos sugieren, desviaban la atención hacia zonas que no eran las de mayor gravedad de la ocultación, sino zonas en donde abundaban los montes, bosques y eriales improductivos, tierras de infimo rendimiento económico, cuya extensión no era fácil de calcular con un mínimo de exactitud y cuya situación jurídica era muchas veces complicada o imprecisa.

Ese tipo de tierras explican que el bloque más compacto de altos índices de ocultación se centrara a ambos lados de la Cordillera Cantábrica (con porcentajes de más del 60 por 100 de ocultación en provincias como León, Santander, Lugo, Oviedo o Burgos), mientras que algunas de las provincias con una constitución social más desigual y oligárquica daban índices por debajo de la media nacional (Cádiz, con un 9 por 100; Sevilla con un 26,4...).

Digo que tales cifras pudieron desenfocar la cuestión del fraude porque el problema no estaba en las vastas extensiones de monte del norte. El problema del fraude no es tanto de cifras absolutas como de desigualdad; cabe imaginar que un reparto extremadamente injusto de los cupos individuales de la Contri- 
bución territorial quedara disimulado por unas cifras globales de riqueza cercanas a las reales (a costa de que algunos contribuyentes figuraran en los amillaramientos con una riqueza superior a la que tenían). Algo así se sospechaba que ocurria en las regiones latifundistas del centro y sur de la península, extremo que el gobierno quiso comprobar descendiendo en ellas a averiguaciones de nivel local, con ayuda de los trabajos topográficos del Instituto Geográfico.

\section{PRIMERAS EVIDENCIAS CARTOGRÁFICAS}

El Instituto Geogräfico emprendió desde 1870 un tipo de trabajo que reviste un gran interés para los historiadores, bajo el nombre de avance catastral. Consistía en realizar mediciones topográficas muy precisas de los términos municipales y de las masas de cultivo, de modo que, comparando las superficies resultantes de sus planos con la suma de superficies declaradas en los amillaramientos, no sólo podría saberse la extensión total de tierras no declaradas, sino además conocer a qué tipo de usos agrícolas correspondian.

Buscando la máxima eficacia propagandística, pero también los menores costes y las mayores bolsas previsibles de ocultación, los topógrafos comenzaron el levantamiento del mencionado avance catastral por la provincia de Córdoba (terminada en 1872), y siguieron por las de Sevilla (1873) y Cádiz (1874). Los trabajos sufrieron un «bache» con la Restauración borbónica, pero el Instituto Geográfico no desapareció — quizá por su vinculación al ejército- y pudo continuar sus trabajos en fechas posteriores: en 1879 se publicaron los datos correspondientes a Albacete, Jaén y Málaga; en 1889 los de Ciudad Real; y en 1893 los de Toledo.

Las cifras de ocultación que se comprobaron en estos trabajos (cuadro 1) confirmaron básicamente la imagen que habian dado las estimaciones del Ministerio de Hacienda en 1870 , con la salvedad de que ahora se contaba sólo con datos de unas cuantas provincias de Andalucía y Castilla la Nueva, pues el avance catastral tardaría más de cien años en cubrir el conjunto del territorio nacional. Si bien las cifras de ocultación comprobadas eran en general inferiores a las que se habian estimado en 1870 , un 26,1 por 100 de la superficie sin controlar seguía siendo un porcentaje importante, que alcanza cotas mucho más altas si aumentamos la escala de observación al nivel provincial (hasta demostrar la ocultación de un tercio de la superficie en las tres provincias del valle del Guadalquivir), o más aún si llevamos la observación hasta el nivel local (con 16 pueblos en los que se venia ocultando impunemente mas de un 80 por 100 de la superficiel). 


\section{CUADRO 1}

Ocultación descubierta en los amillaramientos a raiz de los trabajos del Instituto Geográfico (1872-1893)

\begin{tabular}{|c|c|c|c|c|}
\hline & $A$ & B & $C$ & $D$ \\
\hline Córdoba (1872). & 476.170 & 34,6 & 98,9 & $-10,3$ \\
\hline Sevilla $(1873) \ldots \ldots \ldots \ldots \ldots \ldots \ldots$ & 460.291 & 32,7 & 92,5 & $-72,3$ \\
\hline Cádiz (1874) $\ldots \ldots \ldots \ldots \ldots \ldots \ldots$ & 78.111 & 10,6 & 78,9 & -62 \\
\hline Málaga (1879) ............. & 210.089 & 28,8 & 91,7 & $-46,4$ \\
\hline 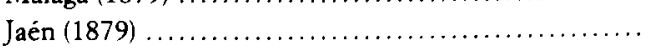 & 451.216 & 33,4 & 76,8 & $-33,1$ \\
\hline Albacete $(1879) \ldots \ldots \ldots \ldots \ldots \ldots \ldots . . . . . . .$. & 477.918 & 32,1 & 100 & $-44,5$ \\
\hline 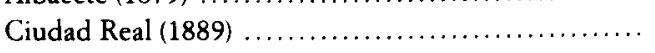 & 377.145 & 19,1 & 87,5 & $-134,6$ \\
\hline 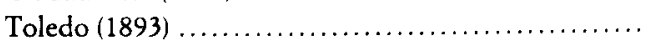 & 238.024 & 15,5 & 90,6 & -108 \\
\hline TOTAL & 2.768 .964 & 26,1 & 100 & $-134,6$ \\
\hline
\end{tabular}

Columna A: Superficie oculta (en hectáreas). Columna B: Porcentaje medio de ocultación de la provincia. Columna $C$ : Porcentaje máximo de ocultación correspondiente a un pueblo de la pro. vincia. Columna $D$. Porcentaje minimo de ocultación correspondiente a un pueblo de la provincia.

FUENTE: Instituto Geográfico: Trabajos topográficos. Comparación de las superficies dadas por el amillaramiento con las que resultan de los planos, Biblioteca del Instituto Geogräfico Nacional, 50.085-50.101.

\section{LA PROVINCIA DE CÁDIZ: LA OCULTACIÓN AL DESCUBIERTO}

Vamos a tomar como modelo del funcionamiento del fraude en la Contribución territorial una de aquellas provincias catastradas en el último tercio del siglo XIX. Para asegurarnos de que no estamos estudiando un caso excepcional, observaremos la provincia de Cádiz, que fue la que arrojó un menor porcentaje de superficie oculta: sus amillaramientos eran, pues, aparentemente, de los más fiables de España, con alrededor de un 10 por 100 de error según las estimaciones de 1870 y según las comprobaciones de 1874 . El cuadro 2 muestra el volumen de ocultación detectado en cada pueblo de la provincia, ordenados de mayor a menor porcentaje de fraude.

Un cuadro como éste resulta muy revelador: descubrimos que, bajo la opaca frialdad de las «medias» aritméticas, las estadísticas provinciales sobre ocultación de tierras en los amillaramientos encerraban una realidad mucho mas desigual de lo que habiamos pensado. Ese supuesto 10,6 por 100 de tierras ocultas, efectivamente, debia de significar bien poco para los habitantes de pueblos como Alcalá del Valle o San Fernando, en donde se venía tributando 


\section{CUADRO 2}

Ocultación descubierta en los amillaramientos de la provincia de Cádiz (1874)

\begin{tabular}{|c|c|c|c|c|}
\hline & $A$ & $B$ & C & $D$ \\
\hline Alcalá de los Gazules & 47.851 & 10.088 & 37.763 & 78,9 \\
\hline Setenil ............... & 8.244 & 4.199 & 4.045 & 49,0 \\
\hline Puerto Real & 19.511 & 10.376 & 9.135 & 46,8 \\
\hline San Roque .... & 16.342 & 8.832 & 7.510 & 45,9 \\
\hline Castellar ..... & 17.913 & 11.427 & 6.486 & 36,2 \\
\hline Trebujena....$\ldots \ldots \ldots$ & 6.983 & 4.562 & 2.421 & 34,6 \\
\hline Chiclana de la Frontera & 20.300 & 13.352 & 6.948 & 34,2 \\
\hline Los Barrios,$\ldots \ldots \ldots \ldots$. & 32.818 & 22.191 & 10.627 & 32,3 \\
\hline Benaocaz .... & 6.929 & 4.966 & 1.963 & 28,3 \\
\hline Algar $\ldots \ldots \ldots \ldots \ldots \ldots$ & 2.686 & 2.037 & 649 & 24,1 \\
\hline Sanlúcar de Barrameda. & 17.095 & 13.235 & 3.860 & 22,5 \\
\hline Espera $\ldots \ldots \ldots \ldots \ldots \ldots \ldots \ldots \ldots$ & 12.313 & 9.600 & 2.713 & 22,0 \\
\hline Conil $\ldots \ldots \ldots \ldots \ldots \ldots \ldots \ldots \ldots$ & 8.563 & 6.927 & 1.636 & 19,1 \\
\hline El Gastor & 2.775 & 2.337 & 438 & 15,7 \\
\hline Rota $\ldots . .$. & 8.183 & 6.987 & 1.196 & 14,6 \\
\hline Medina Sidonia .......... & 54.654 & 47.036 & 7.618 & 13,9 \\
\hline Jimena de la Frontera ... & 34.524 & 30.532 & 3.992 & 11,5 \\
\hline Arcos de la Frontera. & 52.496 & 46.614 & 5.882 & 11,2 \\
\hline Algeciras $\ldots \ldots \ldots \ldots \ldots \ldots \ldots \ldots \ldots$ & 8.375 & 7.575 & 800 & 9,5 \\
\hline Puerto Serrano $\ldots \ldots \ldots \ldots \ldots \ldots \ldots \ldots$ & 2.217 & 2.014 & 203 & 9,1 \\
\hline Paterna de la Rivera $\ldots \ldots \ldots \ldots \ldots \ldots \ldots \ldots \ldots \ldots$ & 1.400 & 1.273 & 127 & 9,0 \\
\hline Chipiona $\ldots \ldots \ldots \ldots \ldots \ldots \ldots \ldots$ & 3.232 & 2.944 & 288 & 8,9 \\
\hline Bornos ...... & 5.433 & 5.034 & 399 & 7,3 \\
\hline Villamartín .......... & 20.977 & 19.734 & 1.243 & 5,9 \\
\hline Torre Alháquime ... & 1.736 & 1.655 & 81 & 4,6 \\
\hline Olvera ............ & 19.192 & 18.676 & 516 & 2,6 \\
\hline Vejer de la Frontera & 39.644 & 38.912 & 732 & 1,8 \\
\hline 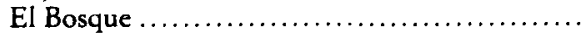 & 3.063 & 3.031 & 32 & 1,0 \\
\hline Villaluenga del Rosario ........................ & 5.988 & 5.936 & 52 & 0,8 \\
\hline Ubrique $\ldots \ldots \ldots \ldots \ldots \ldots \ldots \ldots \ldots$ & 6.936 & 6.887 & 49 & 0,7 \\
\hline Grazalema .... & 12.238 & 12.967 & -729 & $-5,9$ \\
\hline Prado del Rey $\ldots \ldots \ldots \ldots \ldots \ldots \ldots \ldots \ldots \ldots \ldots \ldots \ldots \ldots \ldots$ & 4.959 & 5.445 & -486 & $-9,8$ \\
\hline Puerto de Santa María $\ldots \ldots \ldots \ldots \ldots \ldots \ldots \ldots \ldots$ & 15.640 & 17.954 & -2.314 & $-14,7$ \\
\hline Jerez de la Frontera $\ldots \ldots \ldots \ldots \ldots \ldots \ldots \ldots \ldots$ & 140.461 & 162.866 & -22.405 & $-15,9$ \\
\hline 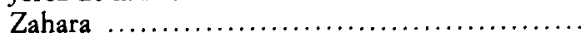 & 7.129 & 8.325 & -1.196 & $-16,7$ \\
\hline 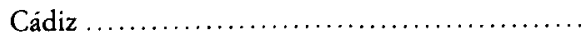 & 942 & 1.108 & -166 & $-17,6$ \\
\hline (n. & 41.455 & 48.818 & -7.363 & $-17,7$ \\
\hline Algodonales .. & 13.599 & 16.216 & -2.617 & $-19,2$ \\
\hline$\ldots \ldots \ldots \ldots \ldots \ldots \ldots \ldots \ldots \ldots$ & 2.831 & 3.941 & -1.110 & $-39,2$ \\
\hline Alcalá del Valle $\ldots \ldots \ldots \ldots \ldots \ldots \ldots \ldots \ldots \ldots \ldots$ & 4.685 & 7.592 & -2.907 & $-62,0$ \\
\hline TOTAL & 732.312 & 654.201 & 78.111 & 10,6 \\
\hline
\end{tabular}

Columna A: Superficie del pueblo en hectäreas, según las mediciones topográficas del Instituto Geográfico. Columna B: Superficie útil declarada en los amillaramientos. Columna $C$ : Ocultación aparente (diferencia A - B). Columna D. Porcentaje de ocultación.

Fuente: Instituto Geográfico y Estadístico: Trabajos topográficos. Provincia de Cádiz (1874). Biblioteca del Instituto Geográfico Nacional, 50.088 . 
por una superficie que excedía en un 62 y en un 39,2 por 100, respectivamente, de la extensión real del término. Efectivamente, en diez pueblos (la cuarta parte de la provincia) se comprobó que los amillaramientos recogian extensiones significativamente mayores que el total del territorio municipal, medido por rigurosos métodos topográficos. Sólo con este dato sería suficiente para despertar nuestra sospecha de que los amillaramientos no se hacían para reflejar realmente la distribución de la propiedad, sino que eran una ficción documental con un trasunto político.

La situación de la provincia de Cádiz no es en absoluto excepcional con respecto a otras provincias españolas, en donde los mismos fenómenos se daban en mayor o menor proporción, como demuestran las columnas $C$ y $D$ del cuadro 1 , en las cuales quedan reflejados los enormes márgenes de variación del porcentaje de tierras ocultas que había dentro de una misma provincia. Junto a esos pueblos «sobrecargados» con más tierras de las que realmente tenían, en la parte alta del cuadro 2 encontramos la otra cara de la moneda: esos pueblos en los que se había ocultado con éxito un 78,9 por 100 de la superficie (Alcalá de los Gazules) o un 49 por 100 (Setenil). Los porcentajes son suficientemente expresivos, pero las cifras absolutas nos ayudan a calibrar la magnitud de la ocultación, comprendiendo que no podía ser sólo fruto de errores de apreciación o de la tolerancia hacia un disimulo fiscal espontáneo: en Alcalá de los Gazules faltaban 37.763 ha, en Jerez sobraban 22.405, en Los Barrios faltaban otras 10.627 ...

¿Qué quiere decir esto? El rasgo que salta a la vista es que, a medida que vamos ampliando la escala de observación, es decir, que nos concentramos en el estudio de territorios más pequeños, la cuantía y la gravedad del fraude aumentan. La existencia de desproporciones tan enormes en el esfuerzo fiscal como las que separaban a unos pueblos y otros dentro de una misma provincia nos acerca al contenido esencial del problema del fraude, que no es sólo un problema de insuficiente recaudación, sino fundamentalmente un problema de desigualdad en el reparto de la carga fiscal, un problema de desigualdad en el acceso a los recursos.

Los trabajos del Instituto Geográfico nos permiten, además, conocer en qué tipo de cultivos se centraban las ocultaciones de tierras, desterrando de una vez por todas la benévola suposición de muchos historiadores según la cual esas enormes cifras ocultas corresponderían en su mayor parte a tierras de poco valor del tipo de montes, prados, yermos y baldíos. Nada de eso: se ocultaban todo tipo de tierras, también tierras de cultivo, y cuanto más valor tenían, más propensos eran sus dueños a hacerlas desaparecer del amillaramiento $o$ a clasificarlas como tierras de rendimiento menor. 
Las cifras del cuadro 3 son suficientemente expresivas de lo que venimos diciendo. Las mayores ocultaciones en cifras absolutas se dan en las tierras ce. realeras de secano. En las tierras de montes y pastos, como era de esperar, también se da una ocultación importante, con más de 28.000 ha de defecto; pero se trata - al mismo tiempo- del tipo de tierras en donde la ocultación supone un porcentaje menor, tan sólo del 8,9 por 100 , por debajo de la media de la provincia. No se sostiene la teoría de que el fenómeno de ocultación de superficies que reflejan las estadisticas procede de las vastas extensiones de montes y pastos cuya medida exacta se ignoraba o cuya titularidad era confusa; por el contrario, la existencia de grandes extensiones de montes y pastos, cuya carga tributaria era infima y no importaba demasiado declararlas, ha sido un factor que ha rebajado las cifras globales del fraude en la Contribución territorial, haciendo que el problema aparente no tuviera las dimensiones del problema real.

\section{CUADRO 3}

Clasificación de las tierras ocultas según su uso. Provincia de Cádiz (1874)

\begin{tabular}{|c|c|c|c|}
\hline & $A$ & $B$ & $C$ \\
\hline Regadio ....... & 4.527 & 2.171 & 47,9 \\
\hline Secano (cereal) .............. & 326.562 & 70.279 & 21,5 \\
\hline Viña $\ldots \ldots \ldots \ldots \ldots \ldots \ldots \ldots$ & 20.160 & 4.970 & 24,6 \\
\hline Olivar $\ldots \ldots \ldots \ldots \ldots \ldots$ & 20.822 & 10.863 & 52,1 \\
\hline 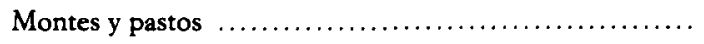 & 321.194 & 28.792 & 8,9 \\
\hline 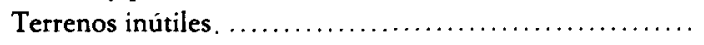 & 29.425 & -19.822 & $-67,3$ \\
\hline
\end{tabular}

Columna A: Extensiones de los cultivos en hectáreas, según los planos del Instituto Geográfico y Estadístico. Columna B: Diferencia con respecto a las superficies declaradas en los amillaramientos. Columna C: Porcentaje de tierras ocultas.

FUENTE: Instituto Geográfico y Estadístico: Trabajos topográficos... Cádiz (1874).

Las cifras de ocultación en montes y pastos vienen determinadas por casos como el de Jerez de la Frontera, en cuyo amillaramiento figuraban 55.585 ha de dehesas, cuando los expertos del Instituto Geográfico no admitieron más que 5.928 (el resto eran clasificaciones discutibles de tierras de otro tipo, en cantidad de $49.930 \mathrm{ha}$ ); o el de Tarifa, en donde se declaraban en los amillaramientos 24.104 ha de dehesas, que luego resultaron ser sólo 4.036; o el de Ve- 
jer de la Frontera, donde de las 7.968 ha de «eriales con pastos» declaradas, los topógrafos no hallaron más que 213 que pudieran recibir tal nombre (las 7.755 restantes daban un amplio margen para ocultar aquí todo tipo de tierras). Ciertamente, las tierras de dehesa se prestaban como ninguna otra categoría a la duda y a la discusión sobre su clasificación fiscal, dadas las características de estas explotaciones en las que se mezclan diversos tipos de aprovechamientos y con muy desigual intensidad; de ahí la facilidad con la que se hacian pasar como «dehesas para pastos» de muy bajo rendimiento terrenos arbolados o cultivados a los que en justicia correspondía tributar por tipos mayores.

Por lo que respecta a las tierras cultivadas, las de olivar y las de regadio parecen ser las más propensas al fraude, pues cerca de la mitad de estas clases habian escapado a los amillaramientos. Las cifras globales encubren situaciones locales muy diferentes, que revelan la desigual fuerza que tenían en cada junta pericial los poseedores de este tipo de tierras. Descendiendo a ese nivel local, encontramos tal volumen de ocultación que es impensable que no fuera conocido por todos: en Vejer de la Frontera se habian evadido de los amillaramientos 3.094 ha de olivar (más de la mitad del total que había en la localidad); y en Villamartín 2.403 ha (las tres cuartas partes de los olivos del pueblo).

Precisamente en este caso de Villamartín resulta muy claro el funcionamiento concreto del fraude. En su amillaramiento aparecen declaradas 825 ha de olivar y 1.838 de dehesas para pastos; pero los topógrafos del Instituto Geográfico midieron 3.228 ha de olivar y sólo 300 de dehesas para pastos. Las 2.403 ha de olivar "perdidas» se encuentran, asi, entre las 1.537 ha declaradas de más como dehesas y las 1.242 de ocultación global en el conjunto del término. Si nos quedamos sólo con este último dato, que supone una ocultación del 5,9 por 100 , apenas percibimos el fraude que se oculta bajo las grandes cifras de un impuesto de cupo, pues la parte de la superficie que se declaraba era objeto de una intensa manipulación a la hora de clasificarla por usos del suelo, manipulación, como vemos, nada desinteresada.

En las tierras de viña el fraude parece haber sido menor; pero una vez más, la cifra media resulta engañosa: las cinco mil hectáreas ocultas sumarian sólo una cuarta parte de las viñas de la provincia (lo cual ya es bastante). Pero si nos concentramos sobre las grandes poblaciones vitivinicolas, en donde los intereses ligados a la viña tenían mayor influencia sobre las instituciones locales, vemos que este cultivo era motivo para grandes bolsas de fraude: en Jerez de la Frontera las mediciones topográficas sacaron a la luz 3.054 ha de viña ocultas (un 35,3 por 100 del total); en Sanlúcar de Barrameda, 1.007 ha $(30,4$ por 100$)$. 
Las tierras de sembradura de secano marcan el índice de ocultación más bajo de las tierras cultivadas, con ese 21,5 por 100 que, no obstante, representaba 70.279 ha que se habian escapado al control de la Hacienda durante un cuarto de siglo. Hay que advertir, sin embargo, que esta menor ocultación aparente en secano que en regadío no demuestra nada parecido a una mayor «honestidad» de sus propietarios, sino que pone de relieve un mecanismo de fraude específico, que consistía en clasificar como tierras de secano fincas que en realidad eran de regadío, o -más en general- hacer que se clasificaran las tierras en una clase de menor rendimiento que el que les correspondia. Un fenómeno parecido puede haber actuado sobre las tierras de pasto y monte, cuyo porcentaje puede ser artificialmente bajo, debido a que en esa categoria se venian escondiendo tierras de cultivo para disfrutar de una carga tributaria más baja.

Llegamos, por último, al difícil apartado de las «tierras inútiles». Los firmantes del trabajo topográfico de 1874 - Carlos Ibáñez, Francisco Vallduví y Andrés de Modet - advirtieron expresamente que en los estados de los amillaramientos no figuraban, por definición, las superficies correspondientes a cascos de poblaciones, cauces de ríos, etc. Sin embargo, esta circunstancia no puede tomarse como atenuante de la gravedad de la ocultación detectada por las comparaciones entre el plano topográfico y los amillaramientos: hemos sumado la extensión total correspondiente a poblaciones, rios, arroyos, barrancas, lagunas, caminos, ferrocarriles, cañadas, marismas, arenales, salinas, esteros, caños, playas, cementerios, cuarteles, arsenales, castillos, zonas militares, muelles, canteras, minas y conventos, todo lo que - desde un punto de vista agricola- podría contabilizarse como «tierras inútiles», incluida la «zona neutral» que separaba San Roque del Gibraltar en manos británicas; y todas esas tierras no suman más que 29.425 ha, muy por debajo de las 119.419 ha descubiertas en los 30 pueblos en los que se detectó mayor superficie que la declarada hasta entonces en los amillaramientos.

Por otra parte, los amillaramientos sí contaban con otra categoría de superficies en sus clasificaciones: las llamadas exactamente «inútiles para todo aprovechamiento", que sumaban nada menos que 49.248 ha (¡y eso que excluían poblaciones, ríos y caminos!). Lo que esto nos muestra es que en esa categoria de tierras inútiles se incluían gran cantidad de tierras de las que sí se obtenían rendimientos agropecuarios. El único tipo de tierras en donde los amillaramientos solian mencionar más extensiones que las que había eran estas tierras «inútiles», que no tenían carga fiscal, lo cual es un fraude tan importante como la ocultación pura y simple, a pesar de que ese índice negativo del $-67,3$ por 100 de ocultación pudiera hacer pensar a primera vista en lo contrario. 
Podemos preguntarnos por la lógica que regía esta distribución del impuesto, o más bien la distribución de las cuotas de fraude. Algunos autores, a partir de investigaciones localizadas sobre todo en Valencia y Cataluña han defendido que el grado de concentración de la propiedad podía ser un factor decisivo para determinar las posibilidades de la ocultación, de manera que las regiones de constitución agraria más «igualitaria» coincidirian con las de menor incidencia de la ocultación sobre los amillaramientos ${ }^{7}$. Por otra parte, investigadores de la historia agraria andaluza han comprobado que las grandes propiedades del sur por lo general sí solian aparecer correctamente declaradas en los amillaramientos, quizá por temor a que su posesión se pusiera en duda, mientras que las mayores ocultaciones se darían en la clasificación y valoración de los terrenos ${ }^{8}$. Ambas observaciones apuntan en la línea de «recuperar» los amillaramientos como una fuente válida para la historia de la propiedad de la tierra, minimizando el grado de ocultación que contienen.

Utilizando los datos del avance catastral levantado a partir de 1906 he intentado establecer una correlación entre las dos variables apuntadas, un índice de concentración de la propiedad y el porcentaje de superficie no declarada en los amillaramientos, comprobando que no existe una correlación significativa. Tampoco pudo establecerse ninguna correlación del fraude con otros indicadores de la estructura agraria de las poblaciones. $Y$ es que, para desesperación de los historiadores que utilizan los amillaramientos como fuente histórica, la lógica que determinaba el nivel de fraude admitido en cada población y a cada contribuyente parece ser la lógica del poder local: las redes per. sonales tejidas en torno a las instituciones municipales, provinciales y estatales estaban constituidas por vínculos multiformes, como el parentesco, la amistad, la asociación económica o la fidelidad política. Por esos canales circulaba el poder en sus formas más puras: la información y la influencia. Y la estructura de esos vínculos y de esas redes determinaba la fuerza de cada población ante el Estado para obtener una carga fiscal soportable, así como la capacidad de cada individuo para eximirse total o parcialmente del reparto del impuesto en su localidad. La variedad de situaciones posibles en cuanto a la cohesión de la oligarquia local y sus vinculos con redes de poder más amplias, hace que la geografía del fraude representada en el mapa escape a cualquier explicación racional en términos globales 9

Al no haber ninguna correlación significativa entre la geografía agraria de

7 Azagra (1978), Ferrer, Segura y Suau (1985), Segura (1988).

8 Bernal (1979), pp. 158-164; Mata (1987), vol. II, pp. 88-90.

9 He tratado más ampliamente este aspecto de la cuestión en Pro (1994b); un estudio local en Pro (1994a). 
las provincias estudiadas y las cifras de ocultación que estamos comentando, puede mantenerse la hipótesis de que el dato esencial para la repartición de los cupos de la Contribución territorial eran las redes de relaciones personales de cada contribuyente, que determinaban la capacidad de cada uno para influir sobre la redacción del amillaramiento.

\section{EL FRAUDE A ESCALA LOCAL}

Si fuera cierta nuestra hipótesis de que la gravedad del problema del fraude se acentúa cuanto más reducida es la muestra que observamos, eso significaría que el problema real tenía unas proporciones mucho mayores de todo lo que puedan indicarnos cualesquiera cifras estadísticas agregadas. $\mathrm{Al}$ menos podemos confirmar que el problema rebasaba ampliamente lo que pudiera indicar ese 26,1 por 100 de tierras ocultas comprobado en 1872-1893, o incluso ese 39,3 por 100 estimado en 1870; para ello vamos a llevar la escala de observación al plano local.

Tomaremos como modelo tres pueblos de la misma provincia de Cádiz: aquel en el que se ocultaban más tierras al fisco según los datos de 1874 (Alcalá de los Gazules), el que más se aproximaba aparentemente a una declaración fiel de su extensión en los amillaramientos (Ubrique, con una diferencia de sólo el 0,7 por 100) y el que había declarado una cantidad por exceso más elevada (Jerez de la Frontera). Hay que señalar que Alcalá de los Gazules es doblemente significativo por su carácter extremo, significativo de hasta dónde podian llegar las ocultaciones dentro del sistema de los amillaramientos, ya que no sólo era el pueblo en el que se ocultaban más tierras en términos absolutos, sino también en porcentaje sobre la superficie total del término.

El caso contrario vendría representado por el pueblo homónimo de Alcalá del Valle, que parece haber tenido un amillaramiento «de castigo» por circunstancias que se nos escapan, pero que suponemos relacionadas con la lógica de la política provincial, en la que los notables locales no debían de estar muy bien situados hacia 1860 (fecha en la que se redactaron los amillaramientos). De todas maneras, el caso resulta también ilustrativo de la mecánica del fraude, aunque sus cifras indiquen aparentemente lo contrario: la sobrecarga tributaria de un territorio o de un grupo de contribuyentes es también una práctica fraudulenta por parte de las juntas periciales, y de hecho se trata de la consecuencia más negativa del fraude, pues al ser la Contribución de inmuebles un impuesto de cupo, la evasión efectiva del impuesto por parte de algunos contribuyentes exige que el resto sean presionados a aceptar un cupo mayor que 
el que les correspondía. Si no hemos incluido en el cuadro los datos de Alcalá del Valle es porque muestran únicamente que en todos los tipos de tierras del término el amillaramiento recogía mayores extensiones de las existentes.

El cuadro 4 arroja alguna luz más sobre lo que se esconde detrás de esas cifras que estamos tomando como indicadores de la ocultación fiscal de tierras a nivel local. Incluso en un caso tan escandaloso como el de Alcalá de los $\mathrm{Ga}$ zules (que había ocultado el 78,9 por 100 de su superficie), al descender a la ocultación por cultivos descubrimos que el fraude era mayor de lo que parecía, pues se habian introducido en las categorías de prados y dehesas para pastos 3.739 ha de tierras de otro tipo, presumiblemente tierras de sembradura de cereales en secano, pues en dicha categoría se habían escamoteado nada menos que 16.880 ha.

En cuanto al caso de Jerez de la Frontera, ya hemos anticipado lo engañosa que resulta la circunstancia de que aparezca en el cuadro general como víctima de una estimación excesiva de su superficie bajo el régimen de los amillaramientos. La totalidad de ese fenómeno se debe al efecto de haber declarado 49.093 ha de dehesas para pastos que el Instituto Geográfico no admitió como tales. Se trata, una vez más, de un caso de clasificación «a la baja» de tierras de cultivo, pues la liberalidad mostrada al declarar dehesas daba un margen realmente amplio para hacer desaparecer fiscalmente 6.000 ha de tierras cerealeras, 1.000 de olivar y - sobre todo- 3.000 de viña, que era el cultivo estratégico en la economía local. La circunstancia de que aparentemente Jerez hubiera sido uno de los pueblos perjudicados por los repartos del impuesto (en virtud de haberle supuesto más superficie de la que tenía) no pasó inadvertida para los notables locales; tal circunstancia fue esgrimida, junto con otros argumentos, por la Cámara agrícola de la localidad en la campaña que impulsó a finales de siglo contra «la enormidad de la tributación con que preténdese estrechar más y más la ya exigua riqueza de las clases productoras» ${ }^{10}$.

Por último, el ejemplo de Ubrique nos sirve para disolver definitivamente el espejismo de pensar que algunos pueblos hubieran podido quedar al margen del movimiento generalizado de ocultaciones masivas al fisco. Bajo esa aparente honestidad que supone el haber declarado una extensión similar a la que luego se comprobó que medía el término, lo que encontramos es que aquella circunstancia parece haber sido fruto de la casualidad. Si nos concentramos sobre las grandes cifras, vemos que la ocultación de tierras cerealeras y de viña (casi $2.000 \mathrm{ha}$ ) queda disimulada por el exceso de superficie encontrado en lo que respecta a dehesas, así como las mil hectáreas de montes no de-

10 Anónimo (1899), p. 159. 


\section{CUADRO 4}

\section{Clasificación de las tierras ocultas según su uso en tres pueblos de la provincia de Cádiz (1874)}

\begin{tabular}{|c|c|c|c|c|}
\hline & $A$ & $B$ & C & $D$ \\
\hline \multicolumn{5}{|l|}{ Alcalá de los Gazules } \\
\hline Regadióo $\ldots \ldots \ldots \ldots \ldots \ldots$ & 112 & 14 & 98 & 88 \\
\hline Secano (cereal) $\ldots \ldots \ldots \ldots \ldots \ldots \ldots \ldots \ldots$ & 19.255 & 2.375 & 16.880 & 87,6 \\
\hline Viña $\ldots \ldots \ldots \ldots \ldots \ldots \ldots \ldots \ldots \ldots \ldots \ldots \ldots$ & 92 & 25 & 67 & 72,8 \\
\hline 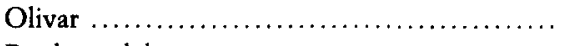 & 363 & 81 & 282 & 77,6 \\
\hline Prados y dehesas ...... & 1.869 & 5.608 & -3.739 & -200 \\
\hline Montes y baldios $\ldots \ldots \ldots \ldots \ldots \ldots \ldots \ldots \ldots$ & 24.624 & 1.477 & 23.146 & 94 \\
\hline Terrenos inútiles $\ldots \ldots \ldots \ldots \ldots \ldots \ldots \ldots \ldots \ldots$ & 1.520 & 506 & 1.014 & 66,7 \\
\hline TOTAL & 47.851 & 10.088 & 37.763 & 78,9 \\
\hline
\end{tabular}

Jerez de la Frontera

Regadio . 149

Secano (cereal) $\ldots \ldots \ldots \ldots \ldots \ldots \ldots \ldots \ldots, \quad 71.161$

Viña 8.629 149 64.382 5.575

Olivar 2.216

Dehesas 6.342

Montes 48.646

Terrenos inútiles 3.315

TOTAL

140.461

1.257

55.435

33.047

2.517

$\begin{array}{rc}0 & 0 \\ 6.779 & 9,5 \\ 3.054 & 35,3 \\ 958 & 43,2 \\ -49.093 & -774,1 \\ 15.598 & 32 \\ 798 & 24 \\ -22.405 & -15,9\end{array}$

\section{Ubrique}

Regadío

$\begin{array}{rrrr}36 & 47 & -10 & -28,6 \\ 3.098 & 1.241 & 1.857 & 59,9 \\ 271 & 211 & 60 & 2,2 \\ 59 & 66 & -6 & -11,8 \\ 0 & 1.279 & -1.279 & - \\ 3.337 & 2.795 & 1.042 & 31,2 \\ 133 & 1.747 & -1.614 & -1.213,5 \\ 6.936 & 6.887 & 49 & 0,7\end{array}$

Columna A: Extensiones de los cultivos en hectáreas, según los planos del Instituto Geografico y Estadístico. Columna $B$ : Extensiones declaradas en los amillaramientos. Columna $C$ : Diferencia A - B. Columna D. Porcentaje de tierras ocultas.

FUENTE: Instituto Geográfico y Estadistico: Trabajos topográficos... Cádiz (1874). 
claradas podrian haber quedado escondidas en esas 1.614 ha de «tierras inútiles para todo aprovechamiento" que nunca existieron. Como se ve $-\mathrm{y}$ salvo por la excepción relativa al regadio, que afecta a una extensión muy pequeña- el movimiento general consiste en declarar las tierras (quizá por el temor a que su titularidad fuera algún día puesta en duda), pero forzando su clasificación en categorías en donde Hacienda apenas podía gravarlas, del tipo de pastos y montes.

\section{SOBRE LA DESIGUAL FIABILIDAD DE LOS DATOS ESTADISTICOS}

Todas las observaciones basadas sobre la comparación de las cifras de los amillaramientos de 1860 con los levantamientos topográficos de 1874 podrian resultar discutibles si entre las dos fechas mencionadas hubiera existido en la provincia estudiada un vasto movimiento de expansión agricola, que hubiera conllevado la roturación de tierras de monte y dehesa para convertirlas en tierras de cultivo. Eso no explicaría la ocultación pura y simple de grandes superficies, ni la extremada desigualdad entre el trato fiscal recibido por los contribuyentes de unos términos y los de otros términos vecinos; pero sí podría explicar algunas de las clasificaciones de tierras cultivadas como pastos y bosques, que nosotros hemos considerado fruto del fraude. Cabe pensar - por ejemplo- que la escasa ocultación detectada en las tierras de pasto y monte, al tiempo que se descubrian grandes extensiones sin declarar de tierras de cultivo, no fuera sólo fruto del fraude, sino de una ampliación real de la superficie cultivada a costa de tierras marginales. Ciertamente, este fenómeno parece haber existido en la segunda mitad del siglo XIX, en parte como consecuencia de la desamortización ${ }^{11}$.

En efecto, hay que señalar que los amillaramientos se pensaron para hacerse cada diez años, pero con un sistema de apéndices anuales que irían adecuando el reparto de los cupos a la evolución agrícola de cada localidad; había, pues, obligación de los ayuntamientos de recoger las declaraciones de modificaciones que hubieran sufrido las propiedades rústicas, tanto en cuanto a su titularidad

11 Asi lo indican una serie de trabajos recientes, como el realizado por González de Molina y Sevilla (1991); no deja de ser llamativo, sin embargo, que gran parte de su razonamiento se apoye sobre las cifras resultantes de los amillaramientos, precisamente en el periodo estratégico de su revisión en torno al cambio de siglo. Este empleo de la fuente ya habia aparecido en trabajos como el de Piqueras (1981), que toma el amillaramiento de 1862 como punto de partida para cuantificar la expansión del viñedo valenciano hasta finales del siglo XIX. 
como en cuanto a su dedicación. El fraude, por lo tanto, existiría incluso cuando se hubiera producido un cambio de uso del suelo, siempre que propietarios y autoridades locales hubieran omitido la notificación del cambio; sabemos que esta era una práctica generalizada. No sólo los apéndices anuales no solian hacerse, sino que nadie declaraba cambio ninguno en el amillaramiento una vez terminado, y además los amillaramientos no se rehicieron con la periodicidad que se había previsto: después de su implantación en 1850, sólo se volvieron a hacer con carácter general en torno a 1860-1864, mientras que todos los intentos posteriores o bien fracasaron (porque contribuyentes y ayuntamientos negaron su colaboración) o bien se realizaron sólo en algunas zonas.

Aun asi, creo que es importante recalcar - considerando de nuevo las cifras generales de la provincia que veíamos en el cuadro 3- que la posibilidad de un cambio significativo en la dedicación de la tierra afectaría principalmente al olivar. Efectivamente, parece que los años 1860-1874, a los que nos estamos refiriendo, coinciden con un proceso de roturación de tierras para plantar olivos, lo cual explicaria la cifra extraordinariamente alta de aparente ocultación en este cultivo $(52,1$ por 100$)$ y la correlativa modestia de la ocultación detectada en cuanto a pastos y montes $(8,9$ por 100$)$. Digo esto siguiendo las indicaciones de uno de los principales especialistas en historia del olivar, que presenta «las décadas centrales del siglo XIX definidas por una importante expansión del cultivo consecuencia, sin duda, de una coyuntura favorable y de numerosos predios por roturar» 12 .

En concreto, la cifra de 10.442 ha de olivar que da Zambrana para la provincia de Cádiz en 1858 la toma de los datos de la Comisión de Estadística publicados en aquella fecha, tan sólo un año después de su fundación y sin haber realizado trabajos de medición topográfica sobre el terreno. Como suele ocurrir en estos casos, se obvia una crítica seria de la forma en que se realizó dicho cálculo, en el que a mi entender iba ya asimilado el efecto de un persistente fraude fiscal. De ser cierta esa cifra, significaría que la extensión dedicada a olivos en Cádiz se habría duplicado en el transcurso de quince años, mientras que en la vecina provincia de Sevilla tan sólo se produjo un incremento de la extensión del olivar del 15 por 100 . Zambrana no se plantea el problema, omitiendo de su comentario la provincia de Cádiz; cuando comenta el caso de Córdoba, en el que se habría dado un incremento similar según sus datos, le parece tan inverosimil que dice escuetamente: «creo que los datos de 1858 son inferiores a la realidad y de ahí el importante avance registrado» ${ }^{13}$.

12 Zambrana (1987), p. 53.

13 Ibidem, p. 57. 
Este tipo de usos de las cifras no es exclusivo del autor citado ni de su obra (por lo demás muy estimable). Es sólo un reflejo de la debilidad de las bases estadísticas sobre las que trabaja la historia económica en España. En concreto, las exageradas distorsiones detectadas en los amillaramientos del siglo XIX nos llevan a poner en cuestión muchas otras fuentes estadísticas que tomaron aquellos documentos como referencia o punto de partida ${ }^{14}$. Ciertamente, los ingenieros agrónomos sospechaban de los amillaramientos y los tomaban con cautela a la hora de evaluar las superficies cultivadas y las producciones agrícolas; pero el nivel de los escrúpulos de cada ingeniero era forzosamente desigual y, en todo caso, el resultado no pasaba de ser una estimación personal, basada sobre pocos datos que podamos considerar rigurosamente objetivos, al menos en los periodos iniciales en torno al cambio de siglo ${ }^{15}$.

En consecuencia de todo lo dicho, creo que la visión optimista sobre el crecimiento de la agricultura española a partir de finales del siglo XIX está viciada por un proceso de mejora progresiva de la calidad de los datos, al ritmo del levantamiento del catastro en esas mismas fechas. Las cifras de partida de muchas series podrian ser artificialmente bajas, no sólo debido a la inexperiencia y las equivocaciones iniciales de los servicios estadísticos, sino también a la ocultación de riquezas, propiedades y rendimientos por temor a su posible empleo fiscal 16

\section{LOS LEVANTAMIENTOS CATASTRALES Y LA EVIDENCIA DE LA OCULTACIÓN}

Los trabajos catastrales realizados en los tres primeros decenios del siglo $\mathrm{XX}$ sirvieron para poner al descubierto de forma cada vez más clara la persis-

34 La treintena de artículos reunidos por Comin y Zafra en El fraude en la bistoria de España (1994) indican que probablemente las dudas sobre la fiabilidad de los amillaramientos puedan extenderse a otras fuentes fiscales y estadisticas; la confianza inconsciente que generan las cifras impresas va dejando paso a la critica tan pronto como se comienza a investigar el modo de producción de los datos.

15 El GEHR (1991, pp. 27-56) ha realizado un análisis detallado sobre lo que se sabe de la forma de elaborar las estadísticas de producción agrícola antes de la Guerra civil. Desgraciadamente, y a pesar de la acumulación de evidencias de sentido contrario, dicho análisis les conduce a concluir con una reivindicación de la bondad de las estadísticas agrícolas españolas (pp. 56-62).

${ }_{16}$ Me refiero a la constatación general de una expansión de la superficie cultivada y de la producción agrícola en la segunda mitad del siglo xIX y el primer tercio del xx, que resulta de la lectura de trabajos tan importantes como los de Garrabou y Sanz (1985), Jiménez Blanco (1985 y 1986), Zapata (1986) y los diversos artículos del GEHR. Coincido en la critica a esta interpretación de la fuente con la postura mantenida por López Estudillo (1989) para el sistema cereal en el período 1891-1935. 
tente ocultación fiscal que practicaban los terratenientes españoles. El mapa 2 muestra los datos agregados de las provincias en las que se realizaron tanto los primeros trabajos del catastro por masas de cultivo y clases de terreno en 1895-1906 como los posteriores del avance catastral en 1906-1924. El panorama de fraude que refleja nos resulta ya tristemente familiar: el sueño de los conservadores de conseguir una sociedad inmóvil parece plasmarse con total eficacia en este terreno de la tributación, tan relevante para la estructuración de la sociedad.

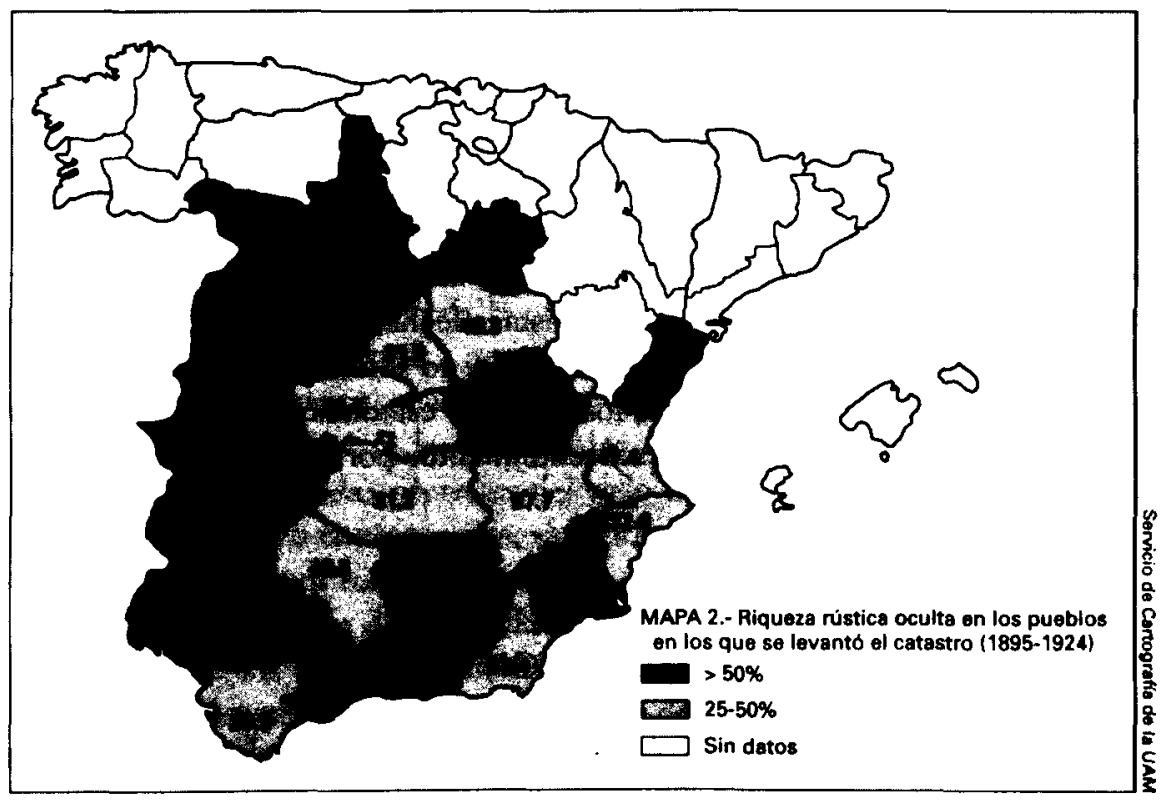

FuENTE: Dirección General de Contribuciones, Impuestos y Rentas (1906); Ministerio de Hacienda (1925).

Los datos del mapa 2 siguen presentando volúmenes de ocultación muy elevados, que en 17 provincias superan el 50 por 100; pero en este caso se trata ya de cifras de riqueza oculta (evaluada por los peritos agrícolas e ingenieros agrónomos a partir de los trabajos catastrales), y no de simples cifras de superficies ocultas como en los cuadros anteriores. Este cambio de criterio se debe a la constatación de que la ocultación «absoluta» de fincas a la Hacienda, si bien era una operación frecuente $e$ importante, no era la única forma de fraude, ni quizá siquiera la más representativa: hemos visto que predominaba la tendencia a clasificar los terrenos en categorías fiscales inferiores a las que les corres- 
pondian, fingiendo dedicaciones apenas productivas (esto es, un supuesto de ocultación «relativa»).

Al mismo tiempo que la presentación de los datos tendía a dar un peso creciente al fraude en las valoraciones, la propia práctica de las evaluaciones de los técnicos catastrales también fue cambiando de tono progresivamente. Puede observarse una tendencia a valorar los terrenos cada vez más alta a lo largo de los tres decenios que van desde la puesta en marcha de los primeros trabajos del catastro por masas a título experimental (1895) hasta que la dictadura de Primo de Rivera detuvo el levantamiento del avance catastral (1925). Así, mientras que en los pueblos en los que se levantó el catastro por masas entre 1895 y 1899 se estimó la ocultación en un 39,1 por 100 de la riqueza, en los avances catastrales levantados entre 1906 y 1924 la estimación llegó al 49,7 por 100. El resultado era que a 31 de diciembre de 1923, el Ministerio de Hacienda podía hacer públicos datos según los cuales la riqueza rústica declarada en los amillaramientos era de 241 millones de pesetas, mientras que la comprobada por el catastro ascendía a algo más del doble, 475 millones ${ }^{17}$.

El gráfico 1 confirma la idea de que los primeros trabajos catastrales realizaron estimaciones más conservadoras o más benévolas de la riqueza rústica, sin duda tratando de evitar la confrontación entre la Hacienda Pública y los terratenientes; a partir de 1917 fue cuando el endurecimiento de las evaluaciones se hizo más notorio, y entonces la resistencia de los terratenientes al levantamiento catastral tomó aires de «Fronda» antifiscal. A medida que se extendía por los campos el catastro, ocultar grandes superficies al fisco se volvia una tarea más complicada; las evaluaciones pasaron a ser la única carta que les quedaba a los defraudadores, de modo que dedicaron un gran esfuerzo de organización y de lucha a defender su posibilidad de jugarla. Efectivamente, la campaña de las cámaras agrícolas contra el avance catastral en los años veinte se concentró sobre las acusaciones de falta de rigor contra las evaluaciones catastrales, suponiéndolas fruto de la voracidad fiscal de los agrónomos y de no haber tenido en cuenta que la coyuntura de precios altos de la primera guerra mundial ya habia pasado a la historia ${ }^{18}$. Tenemos sobrados indicios de que tales protestas eran injustificadas, y que simplemente manifestaban la reacción de los grandes terratenientes contra lo que habría significado el fin de su situación fiscal privilegiada ${ }^{19}$.

17 Ministerio de Hacienda (1925).

18 Pro (1992), pp. 275-289.

19 Entiéndase que el «privilegio» consistía en el control del reparto del impuesto, que permitía a los grandes contribuyentes desviar la carga tributaria sobre los menores propietarios. Que 


\section{GRÁFICO 1}

Ocultaciones de riqueza rística descubiertas por el avance

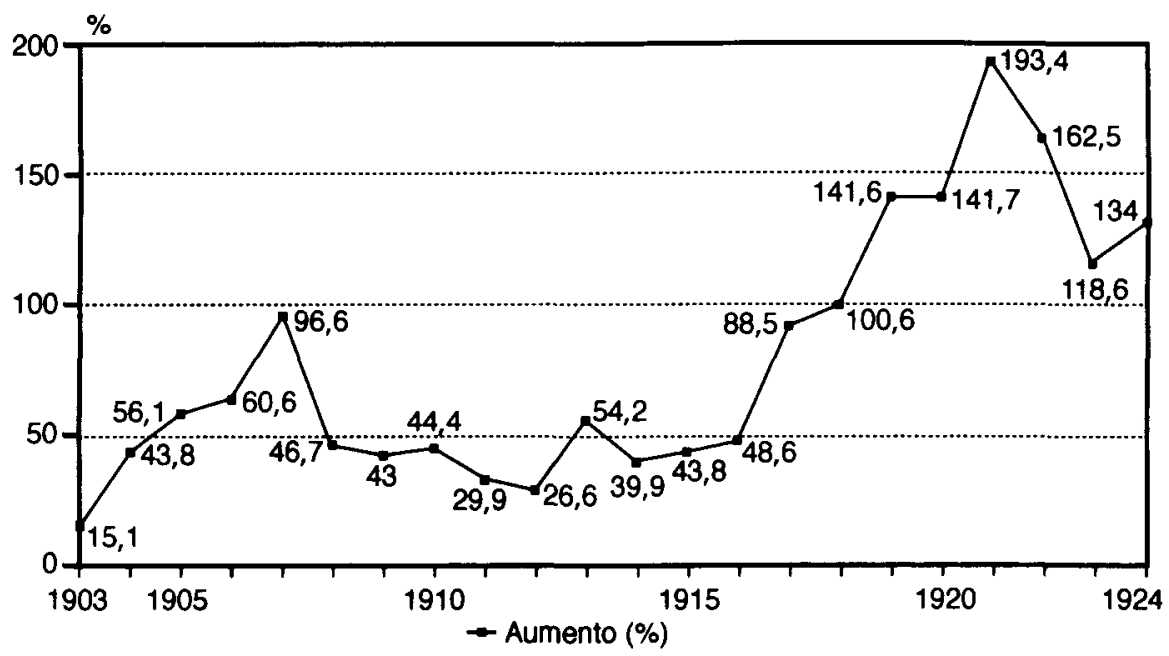

FuentE: Ministerio de Hacienda (1925).

El dato fundamental a retener del mapa 2 y del gráfico 1 es el hecho de que, un siglo después de la reforma de Mon, la ocultación de propiedades rústicas a la Hacienda seguia siendo una realidad muy extendida, gracias a la persistencia del inverosímil sistema informativo de los amillaramientos.

Los trabajos catastrales de principios de siglo permitieron, además, sacar a la luz otro importante dato, como era el del tipo de gravamen al que estaba sometida la riqueza rústica. La cifra media es, en este caso, poco importante, pues depende por completo de la evaluación que se haya querido hacer de las bases del impuesto, evaluación cuya relación con la realidad económica de la época no conocemos. El dato importante es la desigualdad

daría por saber si esa situación de privilegio se mantenia al comparar el esfuerzo fiscal de los terratenientes con el que se exigia a los poseedores de otras formas de riqueza (comerciantes, in. dustriales...). Como es sabido, Fuentes Quintana (1961) ha mantenido que la relativa facilidad de control de la riqueza inmobiliaria se tradujo en una mayor imposición sobre este sector, agravio que cabe pensar que repercutiera en una mayor propensión a ocultar lo que se pudiera y a legitimar este tipo de actitudes. En el estado actual de nuestros conocimientos, sin embargo, nada de esto puede afirmarse, a la espera de que adquieran alguna entidad las investigaciones iniciadas sobre el fraude en los diversos impuestos (como las reflejadas en El fraude fiscal.., 1994). 
de tipos tributarios que un mismo impuesto suponía para distintos contribuyentes.

El cuadro 5 nos muestra los márgenes entre los que se movía la «presión fiscal» por territorial en los distintos pueblos de las provincias para las que tenemos datos. Mientras que el tipo teórico se adaptaba con rigurosa igualdad al 19,6 por 100 que marcaba la ley en aquel momento, la existencia de una gran riqueza oculta hacia que, de hecho, se estuviera tributando, según los pueblos, a tipos medios que iban desde el 4,5 por 100 (en Chiclana, Jaén) hasta el 25,5 por 100 (en Caracuel, Ciudad Real). Si estas son las diferencias entre unos pue blos y otros, no es difícil imaginar que serían mucho mayores las que hubiera entre unos contribuyentes y otros, incluso dentro de una misma localidad. Esta desigualdad de tipos no es ni más ni menos que la consecuencia fiscal práctica del corrupto sistema de los amillaramientos: los amillaramientos, esos documentos que pretendían reflejar la distribución de la propiedad entre los vecinos de un pueblo, eran una ficción adaptada para hacer que esfuerzos fiscales enormemente desiguales tomaran la apariencia exterior de un impuesto proporcional al producto de la agricultura, haciendo que un 4,5 por 100 o un 25,5 por 100 quedaran convertidos en el 19,6 que el ministerio quería, por la magia de la ocultación, de la manipulación sistemática de los datos y de la ne. gociación de las cantidades que las oligarquias terratenientes estaban dispuestas a entregar al Estado.

\section{CUADRO 5}

Variación de los tipos efectivos de la Contribución territorial bajo el régimen de los amillaramientos (1900-1906)

\begin{tabular}{|c|c|c|c|}
\hline & $A$ & $B$ & $C$ \\
\hline Jaén ..... & 9,3 & 4,5 & 16,8 \\
\hline Córdoba & 11,3 & 7,2 & 20,7 \\
\hline Toledo .... & 12,6 & 4,8 & 20,8 \\
\hline Madrid ... & 13,3 & 5,8 & 23,0 \\
\hline Albacete . & 14,1 & 5,0 & 24,9 \\
\hline Ciudad Rea & 15,1 & 7,8 & 25,5 \\
\hline
\end{tabular}

Columna A: Tipo tributario medio de la provincia. Columna B: Tipo medio más bajo detectado en un pueblo de la provincia. Columna $C$ : Tipo medio más alto detectado en un pueblo de la provincia.

FuENTE: Dirección General de Contribuciones, Impuestos y Rentas (1906). 


\section{CONCLUSIÓN}

La magnitud del fraude detectado en los amillaramientos de la propiedad rústica nos permite concluir que tales documentos no son fuentes fiables para el conocimiento de la propiedad y los usos del suelo en la España del siglo XIX. Lejos de constituir meramente una fuente fiscal sesgada «a la baja», los amillaramientos eran fruto de una negociación de los cupos tributarios entre la Administración central y las oligarquías locales, cupos que éstas repartian entre los vecinos según la lógica de los vínculos personales (de parentesco, amistad, patronazgo, etc.).

Durante el primer tercio del siglo xx, con el levantamiento del avance catastral, el Estado fue mejorando paulatinamente su conocimiento de las bases tributarias del impuesto territorial, no sólo porque mejoraron los instrumentos que permitian el control de la riqueza, sino también porque la nueva configuración del poder hizo posible un mayor rigor en los criterios de evaluación. La fiabilidad de las fuentes resultantes nos sirve de término de comparación para poner en evidencia la falsedad de los amillaramientos. Pero, al mismo tiempo, modifica las bases sobre las que debemos estimar el crecimiento de la agricultura española desde la Guerra de Cuba hasta la Guerra civil: la apariencia de un crecimiento espectacular en esos cuatro decenios queda rebajada -en proporciones que ignoramos- al saber que las cifras contienen el efecto de una mejora sustancial en el acercamiento de los servicios estadísticos del Estado a la realidad agrícola.

\section{BIBLIOGRAFIA}

Anónimo (1899): «Tareas de la Cámara», Boletín de la Cámara Agricola de Jerez de la Frontera, núm. 282.

AZAGRA, J. (1978): «Los padrones de riqueza como fuente para la historia agraria del siglo xIX», en Estudios de historia de Valencia, Valencia, pp. 415-432.

BerNal, A. M. (1979): La lucha por la tierra en la crisis del Antiguo Régimen, Madrid.

BESNIER, L. (1964): Medidas y pesos agrarios, Madrid.

Direccion General de Contribuciones, Impuestos y Rentas (1906): Datos relativos al Catastro por Masas de Cultivo y a los Registros fiscales de la propiedad rística, Madrid.

- (1909): Memoria resumen de los trabajos de avance catastral llevados a cabo en la provincia de Albacete, Alicante.

Ferrer, Ll.; Segura, A., y Suau, J. (1985): «Confecció dels amillaraments i poder local: els municipis del Pla de Barcelona, 1851-1874m, en I. Moll, ed.: La vida quotidiana dins la perspectiva historica, Palma de Mallorca, pp. 143-173. 
El fraude fiscal en la bistoria de España (1994), núm. monográfico de Hacienda Pública Española, I.

FUENTES QuiNTANA, E. (1961): «Los principios del reparto de la carga tributaria en España», Revista de Derecho Financiero y de Hacienda Pública, núm. 41, pp. 161-298.

GARCIA DE TORRES, J. (1872): Memoria relativa a los impuestos y servicios centralizados en la Dirección General de Contribuciones, redactada por el director general del ramo, Madrid.

Garrabou, R., y SANZ Fernandez, J. (1985): «La agricultura española durante el siglo XIX: ¿inmovilismo o cambio?», introducción a: R. Garrabou y J. Sanz Fernández, eds.: Historia agraria de la España contemporánea. 2. Expansión y crisis (1850-1900), Barcelona, pp. 7-191.

GEHR (GRUPo de Estudios DE Historia RuRAL) (1983): «Evolución de la superficie cultivada de cereales y leguminosas en España, 1886-1935», Agricultura y Sociedad, núm. 29, pp. 285-325.

- (1984): «El uso del suelo y la producción agraria en España (1891-1931)», Papeles de Economía Española, núm. 20, pp. 66-67.

(1989): «El sector agrario hasta 1935», en A. Carreras, ed.: Estadísticas históricas de España. Siglos XIX y XX, Madrid, pp. 91-129.

- (1991): Estadísticas bistóricas de la producción agraria española, 1859-1935, Madrid.

Gonżalez de Molina Navarro, M., y Sevilla Guzman, E. (1991): «Minifundio y gran propiedad agraria: estabilidad y cambio en la Alta Andalucía, 1758-1930»; en: P. Saavedra y R. Villares, eds.: Señores y campesinos en la Península Ibérica, siglos XVIII-XX. 2. Campesinado y pequeña explotación, Barcelona, pp. 88-138.

INSTITUTO GeOGRÁFICO Y EsTADISTICO (1886): Equivalencias entre las pesas y medidas usadas antiguamente en las diversas provincias de España y las legales del sistema métrico-decimal, Madrid.

Jimenez BlanCO, J. I. (1985): Crisis y expansión de la agricultura de Andalucía oriental, 1874 1936, Madrid.

- (1986): «El nuevo rumbo del sector agrario español (1900-1936). Introducción», en: R. Garrabou, C. Barciela y J. I. Jiménez Blanco, eds.: Historia agraria de la España contemporánea. 3. El fin de la agricultura tradicional (1900-1960), Barcelona, pp. 9-141.

LOPERA, F. (1958): Equivalencias agrarias, métricas, de longitud y de peso, Madrid.

LOPEZ Estudillo, A. (1989): «Notas para una revisión crítica de las estadísticas sobre la producción y rendimientos de cereales y leguminosas entre 1891 y 1935 m, comunicación inédita al IV Congreso de la Asociación de Historia Económica, Alicante.

MATA, R. (1987): Pequeña y gran propiedad agraria en la depresión del Guadalquivir, Madrid.

Ministerio de Hacienda (1925): Resumen numérico de la Memoria formulada por la Sección de Catastro de la Riqueza Rústica. Año de 1924, Madrid.

Piqueras, J. (1981): La vid y el vino en el País Valenciano. Geografía económica: 1564-1980, Valencia.

PRo Ruiz, J. (1992): Estado, geometría y propiedad. Los origenes del catastro en España, 1715 1941, Madrid.

- (1994a): «Fraude, statistique et pouvoir dans l'Espagne libérale (1840-1868)», $R e$ vue d'bistoire moderne et contemporaine, núm. 41-2, pp. 253-268.

(1994b): «El poder de la tierra: una lectura social del fraude en la Contribución de inmuebles, cultivo y ganaderia (1845-1936)», en El fraude fiscal... (1994), pp. 189-201.

Segura, A. (1988): «La reforma de Mon (1845) y los amillaramientos de la segunda mitad del siglo XIX», en El Catastro en España. I: 1714-1906, Madrid, pp. 113-132. 
Sociedad Economica Matritense de Amigos del Pais (1874): Dictamen que presentó la comisión encargada del estudio de la proposición que tiene por objeto llevar a cabo unos nuevos amillaramientos, Madrid.

Tablas de correspondencia recíproca entre las pesas y medidas métricas mandadas utilizar en España por la Ley de 19 de Julio de 1849 y las que actualmente están en uso, Madrid, 1852.

ZambRANA, J. F. (1987): Crisis y modernización del olivar español, 1870-1930, Madrid.

ZaPATA BLANCO, S. (1986): La producción agraria de Extremadura y Andalucía occidental, 1874-1935, Madrid. 